\title{
Can We Source the Origin of the Feral Tankwa Goat of South Africa?
}

Sahar Ahmed ${ }^{1}$, Anna S Kropff ${ }^{2 *}$, Thinus Jonker ${ }^{3}$ and Antoinette Kotzé ${ }^{2,4}$

${ }^{1}$ Department of Cell Biology, National Research Center, Dokki, Giza, Egypt

${ }^{2}$ National Zoological Garden, South African National Biodiversity Institute, P.O. Box 754, Pretoria, 0001, South Africa

${ }^{3}$ Northern Cape Department of Agriculture, Land Reform \& Rural Development, P.O. Box 5018, Kimberley, 8300, South Africa

${ }^{4}$ Department of Genetics, University of the Free State, P.O. Box 339, Bloemfontein, 9300 South Africa

"Corresponding author: Anna S Kropff, National Zoological Garden, South African National Biodiversity Institute, P.O. Box 754, Pretoria, 0001, South Africa, Tel: +27122292781; E-mail: s.kropff@sanbi.org.za

Received date: August 20, 2018; Accepted date: September 25, 2018; Published date: October 01, 2018

Copyright: $\odot 2018$ Ahmed S, et al. This is an open-access article distributed under the terms of the Creative Commons Attribution License, which permits unrestricted use, distribution, and reproduction in any medium, provided the original author and source are credited.

\begin{abstract}
Tankwa goats are feral animals initially identified from the Tankwa Karoo National Park in the Northern Cape, South Africa that is reported to be well adapted to their harsh environment. We assessed the genetic diversity of these goats by sequencing the hypervariable segment of the mitochondrial control region (mtDNA HVI D-loop). We additionally clarified genetic origin of the Tankwa goats relative to international breeds. A total of 40 blood samples were collected between 2012 and 2016 from individual unrelated Tankwa goats. Genomic DNA was extracted and the amplified $m t D N A$ D-loop region was used for phylogenetic analysis. A total of 12 haplotypes were identified. Haplotype and nucleotide diversity of Tankwa goat's population was identified to be low compared to the global goat populations including South African goats, which may be an indication of higher inbreeding in this population. The results obtained from analysis of the $m t D N A$ region in Tankwa goats identified a unique population constructed of two lineages (A $42.5 \%$ and B 57.5\%).
\end{abstract}

Keywords Tankwa goat; Genomic DNA; D-loop

\section{Introduction}

Goats (Capra hircus) were the first animals to be domesticated [1]. In South Africa, goat populations are of cultural, socio-economic and ecological importance. Goats are used as a protein source in low income farming communities; to trade for other goods and as an effective way of preventing bush encroachment in grassland areas [2]. The feral goats initially identified from the Tankwa Karoo National Park in the Northern Cape, South Africa, constitute a unique genetic resource [3]. Indications are that these Tankwa goats have been freeroaming in the park for more than 8 years. Two populations are currently being recognized, the one in the National Park estimated at just over a 100 individuals and the other at the Carnarvon Research Station with more than 300 individuals. The goats are reported to be well adapted to the harsh environment and have had little human intervention such as artificial selection. These goats are parasitetolerant, have good mothering abilities and seem to survive high predation pressures. Current efforts to declare the Tankwa goat as a landrace breed in South Africa by the National Department of Agriculture, Forestry and Fisheries, necessitated the need to investigate and compare the genetic origin and evolutionary relationship with other goat breeds.

The approach we used is based on the maternally inherited mitochondrial DNA (mtDNA) analysis as this may provide evidence of the ancestry of domestic breeds dating back hundreds of generations. Several studies used the Hypervariable region (HVI) of the $m t D N A$ Dloop region to assess phylogenetic relationships and maternal origin of different goat populations. According to the maternal origin, Naderi et al. $[4,5]$ grouped populations around the world into six haplotypes (A, $B, C, D, F \& G)$. Our study aims to determine the genetic diversity in the hypervariable segment of the $m t D N A$ control region ( $m t D N A$ HVI D-loop) and to clarify the genetic origin of the Tankwa Goat.

\section{Materials and Methods}

\section{Samples}

A total of 40 blood samples were collected between 2012 and 2016 from individual unrelated Tankwa goats at the Carnarvon Research Station. Samples were stored at $-20^{\circ} \mathrm{C}$ at the National Zoological Garden, South African National Biodiversity Institute (NZG, SANBI) Biobank until DNA extraction.

\section{Genetic analysis}

Genomic DNA was extracted from whole blood using the GeneJet Genomic DNA Purification kit (Thermofisher Scientific, California, USA) following the manufacturers protocol. Polymerase Chain Reaction (PCR) amplifications were conducted using the CAP-F and CAP-R primers as described by Luikart et al. [6]. Standard PCR protocol was used to PCR the samples and the standard protocol for the BigDye Terminator v3.1 Cycle Sequencing Kit (Thermofisher Scientific, California, USA) was used to sequence the samples. The sequences were visualized using the 3130 Genetic Analyzer (Thermofisher Scientific, California, USA). Sequencing reactions were completed for both the forward and reverse direction. For further information on the PCR and sequencing conditions the authors can be contacted.

\section{Statistical analysis}

Sequences were aligned, inspected, trimmed and exported in FASTA format by using Geneious 6.0 software [7]. The DnaSP software 4.10 
[8] was used to generate a haplotype file, and to identify haplotypes occurring in more than one individual, to ensure that each unique haplotype occurs only once during phylogenetic analysis. The consolidated dataset was used for further analysis. The hierarchical distribution of total genetic diversity was determined using an analysis of molecular variance (AMOVA), as implemented in Arlequin. The population pairwise difference was determined using Arlequin. Seventeen sequences representative of lineages A- F [4] and wild bezoar goat (Capra aegagrus, EF989163 \& EF989426); C. caucasica (AJ317875); C. sibirica (AJ317874); C. cylindricornis (AJ317870); $C$. nubiana (AJ317871) and C. falconeri (AJ317872 \& AB110591) were downloaded from GenBank and added to our dataset as representative of the global domestic and wild goat diversity. The MEGA software 7 [9] was used to construct a Maximum Likelihood tree and to identify the relationships between the Tankwa population and the global goat diversity, with 1000 bootstrap replications. All positions containing gaps and missing data were eliminated. The genetic variation of the population was expressed as haplotype diversity (h), from DNA SP; and nucleotide diversity $(\pi)$ [10], from Arlequin [11]. In addition, fifteen sequences representative of South African goat breeds (AJ317812-15; AJ317819-22; AJ317844; EF618351-56) [6,7] were downloaded from GenBank to compare with the Tankwa goats.

\section{Results}

The hypervariable segment (HVI) of the mtDNA control region of the Tankwa goat indicated a sequence length obtained after alignment by Geneious 8.0 software (excluding sites with gaps / missing data) of 455-bp. The alignment sequences were further used for analysis. The DNA SP software identified 12 haplotypes for the Tankwa goat. These haplotypes were deposited in the GenBank database (Accession numbers: MG602010- MG602021). The haplotype diversity and the average number of nucleotide differences $(\mathrm{k})$ was $0.719 \pm 0.056$ and 11.299 , respectively. The nucleotide diversity $(\pi)$ among the Tankwa goats population was $0.024 \pm 0.001$. The HVI amplified fragment observed polymorphic with 30 variable sites over the 455-bp of the alignment. The variable sites position (two variants) recorded 5 sites at $3,6,131,436$ and 437. The parsimony informative sites position (two variants) recorded 23 sites at 12, 58, 72, 108, 129, 132, 157, 177, 184, $194,213,228,232,240,252,266,270,271,279,287,297,300$ and 305.

Phylogenetic analyses were conducted in MEGA7. The analysis included the Tankwa goat sequences, the GenBank data of the global domestic goat lineages A-F and eight wild goats' references. The Maximum Likelihood method was used for the phylogenetic analysis based on the Hasegawa-Kishino-Yano model (HKY+G+I) as determined by running a modeltest in MEGA 7 [12]. The initial tree for the heuristic search was obtained automatically by applying Neighbor-Join and BioNJ algorithms to a matrix of pairwise distances estimated using the Maximum Composite Likelihood (MCL) approach, and then selecting the topology with superior log likelihood value. The tree is drawn to scale, with branch lengths measured in the number of substitutions per site. The analysis involved 67 nucleotide sequences. All positions containing gaps and missing data were eliminated. The results of the phylogenetic analysis revealed that the Tankwa goat grouped to the A and B lineages (Figure 1). Lineage A (GA_TK) was observed with $42.5 \%$ compared to $57.5 \%$ for lineage B (GB_TK).



Figure 1: The Maximum Likelihood circular phylogram showing Tankwa goat population, 17 reference haplogroups (A-F) and 8 wild Capra. The evolutionary history was inferred.

Figure 2 illustrates the phylogram constructed for the Tankwa goat population using the Maximum Likelihood method based on the Hasegawa-Kishino-Yano model (HKY+G+I) [12]. The analysis involved 40 nucleotide sequences. All positions containing gaps and missing data were eliminated. The final dataset was 455 positions. The construction showed two branches with two main clusters for lineage A and one cluster for lineage B. The results of the Mega7 [9] analysis for the construction of the Phylogram between the Tankwa goat and 15 South African goat sequences downloaded from Genbank, is illustrated in Figure 3. The analysis involved 55 nucleotide sequences. All positions containing gaps and missing data were eliminated. There were a total of 441 positions in the final dataset. The South African goats lineages were divided between the Tankwa goat lineage $\mathrm{A}$ and $\mathrm{B}$ in 12 (GA_SA) and 3 (GB_SA) individuals, respectively.

AMOVA showed that the Tankwa goat lineage A and B included 6 haplotypes for each lineage. Haplotype 1 and haplotype 4 had the highest frequencies of $0.705 \pm 0.113$ (GA_TK) and $0.782 \pm 0.087$ (GB_TK) respectively. Ten haplotypes were identified in the SA goat populations with the highest frequencies observed in haplotype 1 $(0.417 \pm 0.148)$ and haplotype $4(0.333 \pm 0.333)$. Shared haplotypes were observed between GA_TK and GA_SA for haplotype 1 while the haplotype 4 was shared between GB_TK and GB_SA goats (Table 1).

The nucleotide diversity $(\pi)$ of GA_TK and GB_TK was $0.0017 \pm$ 0.0014 compared to $0.0025 \pm 0.002$ and $0.0045 \pm 0.0042$ for GA_SA and GB_SA, respectively. The GA_SA and GB_SA haplotype diversity were $0.833 \pm 0.1002$ and $1.000 \pm 0.272$ respectively compared to 0.515 \pm 0.145 and $0.395 \pm 0.127$ for GA_TK and GB_TK (Table 2).

\begin{tabular}{|c|c|c|c|c|}
\hline Haplotype & GA_TK & GB_TK & GA_SA & GB_SA \\
\hline Hap_1 & $0.705 \pm 0.113$ & --------- & $0.417 \pm 0.148$ & --------- \\
\hline Hap_2 & --------- & $0.043 \pm 0.043$ & -------- & --------- \\
\hline Hap_3 & $0.058 \pm 0.058$ & ---------- & ---------- & ---------- \\
\hline
\end{tabular}


Citation: Ahmed S, Kropff AS, Jonker T, Kotzé A (2018) Can We Source the Origin of the Feral Tankwa Goat of South Africa?. J Phylogenetics

Page 3 of 5

\begin{tabular}{|c|c|c|c|c|}
\hline Hap_4 & -------- & $0.782 \pm 0.087$ & |-------- & $0.333 \pm 0.333$ \\
\hline Hap_5 & --.-- & $0.043 \pm 0.043$ & ----'--' & |----'-- \\
\hline Hap_6 & --------' & $0.043 \pm 0.043$ & |-------- & |-------- \\
\hline Hap_7 & $0.058 \pm 0.058$ & --.-- & |--.--'- & |---.--' \\
\hline Hap_8 & $0.058 \pm 0.058$ & --------- & |-------- & |--------' \\
\hline Hap_9 & $0.058 \pm 0.058$ & -------' & |------- & |------- \\
\hline Hap_10 & --------- & $0.043 \pm 0.043$ & |-------- & |-------- \\
\hline Hap_11 & -------- & $0.043 \pm 0.043$ & |-------- & |-------- \\
\hline Hap_12 & $0.058 \pm 0.058$ & --------- & |-------- & |-------- \\
\hline Hap_13 & --.--- & ------ & $0.083 \pm 0.083$ & |-------- \\
\hline Hap_14 & --------- & --------- & $0.083 \pm 0.083$ & |--------- \\
\hline Hap_15 & ------- & ------- & $0.167 \pm 0.112$ & |-----'- \\
\hline Hap_16 & -------- & -------- & $0.083 \pm 0.083$ & |-------- \\
\hline Hap_17 & --.--- & ----'--' & |------ & $0.333 \pm 0.333$ \\
\hline Hap_18 & --------- & --------- & $0.083 \pm 0.083$ & |--------' \\
\hline Hap_19 & --------- & -------- & $0.083 \pm 0.083$ & |------- \\
\hline Hap_20 & ------- & - & |------- & $0.333 \pm 0.333$ \\
\hline
\end{tabular}

Table 1: Frequencies of haplotypes of Tankwa goat (GA_TK, GB_TK) and 15 South African goat GA_SA and GB_SA).

\begin{tabular}{|l|l|l|l|}
\hline Population & $\begin{array}{l}\text { Number of } \\
\text { haplotypes }\end{array}$ & $\begin{array}{l}\text { Haplotype diversity } \\
\text { (SD) }\end{array}$ & $\begin{array}{l}\text { Nucleotide diversity } \\
\text { (SD) }\end{array}$ \\
\hline GA_TK & 6 & $0.515 \pm 0.145$ & $0.0017 \pm 0.001$ \\
\hline GB_TK & 6 & $0.395 \pm 0.127$ & $0.0017 \pm 0.001$ \\
\hline GA_SA & 7 & $0.833 \pm 0.100$ & $0.0025 \pm 0.002$ \\
\hline GB_SA & 3 & $1.00 \pm 0.272$ & $0.0045 \pm 0.004$ \\
\hline
\end{tabular}

Table 2: Number of haplotypes (h) in Tankwa goats (GA_TK, GB_TK) and 15 South African GA_SA and GB_SA) goats following alignment, haplotype diversity (hd) and nucleotide diversity $(\pi)$, and mean number of pairwise differences (with standard deviation-SD).

\begin{tabular}{|l|l|l|l|l|}
\hline & GA_TK & GB_TK & GA_SA & GB_SA \\
\hline GA_TK & 0 & & & \\
\hline GB_TK & 0.968 & 0 & & \\
\hline GA_SA & 0.031 & 0.963 & 0 & \\
\hline GB_SA & 0.953 & 0.553 & 0.936 & 0 \\
\hline
\end{tabular}

Table 3: Distance pairwise difference between the Tankwa goat and South African goats, calculated Fst values between population pairs, with associated significance $(\mathrm{P})$ values. Significant differentiation at $\mathrm{p}<0.05$.

The pairwise distance between all populations ranged from 0.968 to 0.031 . The distance between GA_TK and GA_SA was 0.031 while it was 0.553 between GB_TK and GB_SA populations. Significant Fst P values at Level $=0.05$ were observed between GA_TK and GA_SA (Table 3).

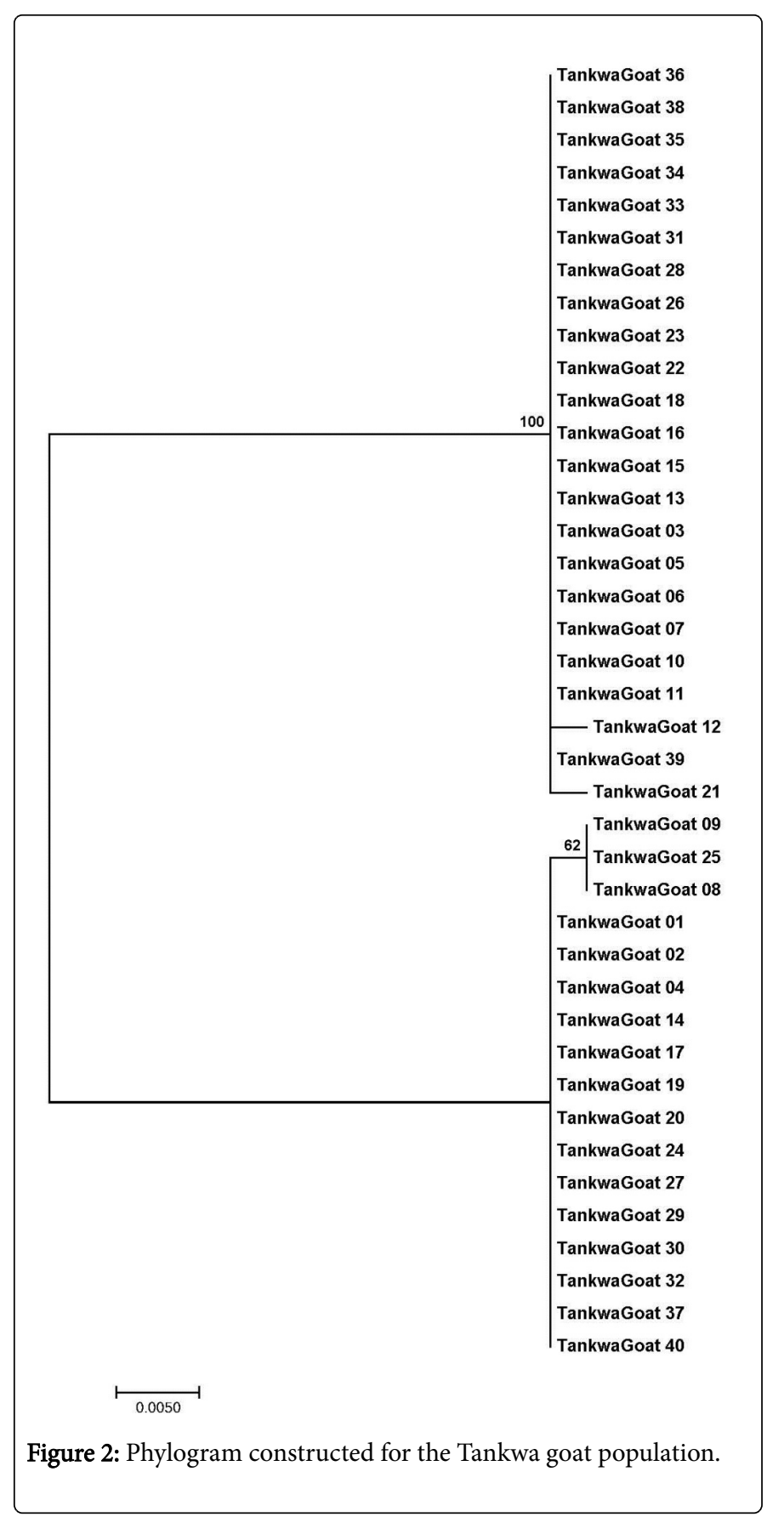






Figure 3: Phylogram constructed for the Tankwa goat population and 15 sequences from South African goats downloaded from GenBank.

\section{Discussion}

Phylogenetic analysis and comparison with the global domestic goats lineages, indicates that the Tankwa goat falls within two lineages (A $42.5 \%$ and B 57.5\%). A total of 12 haplotypes with $0.719 \pm 0.056$ haplotype diversity were identified in the population. The recorded nucleotide diversity of the Tankwa goats population was $\pi=0.0017$ in both lineages (Table 2) wherein the number of bases which are not identical ranged from 0 to 25 bases in the 455 -bp of the amplified MTDNA D-loop. The obtained results revealed that the haplotype and nucleotide diversity of Tankwa goats population are low compared to previous studies of global goat populations [4,6,13-21].

The genetic structure of the Tankwa goat population revealed that lineage $B$ was of a higher frequency compared to lineage A with lineage B being less diverse $(0.395 \pm 0.127)$ compared to lineage A $(0.515 \pm$ 0.145). According to Naderi et al. [4,5] lineage A is the more common haplotype worldwide (more than 90\%), comparing the diversity of Tankwa goat haplotype A to the global goat populations, the results illustrated that the Tankwa goats lineage A showed lower level of diversity $(0.515 \pm 0.145)$ compared to the global goat populations $(0.999 \pm 0.0001)$. The lineage B haplotype diversity in the Tankwa goat was low $(0.395 \pm 0.127)$ compared to the lineage B haplotypes diversity of Chinese goats $(0.879 \pm 0.019$ to $0.961 \pm 0.039)[16,20]$ and those from Laos, Mongolia and South Korea $(0.815 \pm 0.048$ to $0.900 \pm 0.019)$ [4].

The obtained results from comparative the genetic structure of the South African goat populations reported by Luikart et al. [6] and Naderi et al. [7] and the current study of Tankwa goats population investigated provided evidence that the South African goat populations showed higher haplotype and nucleotide diversity compared to the Tankwa goats population. The observed genetic distance of Lineage A between Tankwa goats and the South African goat populations was significant. The results suggested that Tankwa goats are isolated and may be more inbred in comparison to other South African goat breeds.

The global patterns of genomic diversity between wild Bezoar ibex and domestic Iranian and Moroccan goats have been studied by Alberto et al. [1]. They found that the Bezoar ibex showed lower nucleotide diversity than Iranian goats and higher inbreeding than Iranian and Moroccan goats. Their analysis reported that 30 regions in the genome have nucleotide diversity and haplotype clustering between wild and domestic groups. These regions included immunity (14 genes), productivity traits associated to milk composition (11 genes), meat (11 genes), and hair characteristics (4 genes), fertility (2 genes), and neural development, and the nervous system (5 genes). This valuable study indicated the domesticated goats are higher in genetic diversity due to several factors including selection and demographical reasons. The results obtained from Tankwa mtDNA region observed a unique goat's population constructed of two lineages of low haplotypes and nucleotide diversity which may suggest higher inbreeding.

In this study we investigated and compared the genetic origin and evolutionary relationship with other goat breeds. This study identified the genetic origin of the goats; however, these results should be confirmed with additional analysis such as genetic characterization of the population using nuclear DNA markers, Y-Chromosome markers or with the Illumina goat $50 \mathrm{~K}$ SNP panel to determine further evolutionary relationships between these goats and other breeds [22]. 


\section{Disclosure Statement}

The authors declare no conflict of interest.

\section{Funding Information}

This project was funded by the Northern Cape Department of Agriculture, Land Reform \& Rural Development (DALRRD).

\section{Acknowledgments}

We acknowledge the Carnarvon Research Station, the Northern Cape Department of Agriculture, Land Reform \& Rural Development (DALRRD) staff who are committed to the conservation of the Tankwa goat population.

\section{References}

1. Alberto FJ, Boyer F, Orozco-terWengel P, Streeter I (2018) Convergent genomic signatures of domestication in sheep and goats. Nature Communications 9: 83

2. Mahanjana AM, Cronjé PB (2000) Factors affecting goat production in a communal farming system in the Eastern Cape region of South Africa. S Afr J Anim Sci 30: 149-154.

3. Kotzé A, Grobler JP, van Marle-Köster E, Jonker T, Dalton DL (2014) The Tankwa Karoo National Park feral goat population: A unique genetic resource. S Afr J Anim Sci 44: 43-48.

4. Naderi S, Rezaei H-R, Taberlet P, Zundel S, Rafat SA, et al. (2007) Largescale mitochondrial DNA analysis of the domestic goat reveals six haplogroups with high diversity. PLoS One 2: e1012.

5. Naderi S, Rezaei H-R, Pompanon F, Blum MGB, Negrini R, et al. (2008) The goat domestication process infereed from large-scale mitochondrial DNA analysis of wild and domestic individuals. PNAS 46: 17659-17664.

6. Luikart G, Gielly L, Excoffier L, Vigne JD, Bouvet J, et al. (2001) Multiple maternal origins and weak phylogeographic structure in domestic goats. Proc Natl Acad Sci USA 98: 5927-5932.

7. Kearse M, Moir R, Wilson A, Stones-Havas S, Cheung M, et al. (2012) Geneious Basic: An integrated and extendable desktop software platform for the organization and analysis of sequence data. Bioinformatics 28 : 1647-1649.

8. Rozas J, Sanchez-DelBarrio JC, Messeguer X, Rozas R (2003) DnaSP, DNA polymorphism analyses by the coalescent and other methods. Bioinformatics 19: 2496-2497.
9. Kumar S, Stecher G, Tamura K (2016) MEGA7: Molecular evolutionary genetics analysis version 7.0 for bigger datasets. Mol Biol Evol 33: 1870-1874.

10. Nei M (1987) Molecular Evolutionary Genetics. Columbia University Press, New York

11. Excoffier L, Laval G, Schneider S (2005) Arlequin ver. 3.0: An integrated software package for population genetics data analysis. Berne (Switzerland): Institute of Zoology, University of Berne.

12. Hasegawa M, Kishino H, Yano TA (1985) Dating the human-ape splitting by a molecular clock of mitochondrial DNA. J Mol Evol 22: 160-174.

13. Joshi MB, Rout PK, Mandal AK, Tyler-Smith C, Singh L, et al. (2004) Phylogeography and origin of indian domestic goats. Mol Biol Evol 21: 454-462.

14. Liu RY, Gong-She Y, Chu-Zhao L (2006) The genetic diversity of mtDNA D-loop and the origin of chinese goats. Acta Gen Sin 33: 420-428.

15. Liu RY, Lei CZ, Liu SH, Yang GS (2007) Genetic diversity and origin of chinese domestic goats revealed by complete mtDNA D-loop sequence variation. Asian-Aust J Anim Sci 20: 178-183.

16. Liu YP, Cao SX, Chen SY, Yao YG, Liu TZ (2009) Genetic diversity of Chinese domestic goat based on the mitochondrial DNA sequence variation. J Anim Breed Genet 126: 80-89.

17. Pereira F, Pereira L, Van Asch B, Bradley DG, Amorim A (2005) The mtDNA catalogue of all Portuguese autochthonous goat (Capra hircus) breeds: High diversity of female lineages at the western fringe of European distribution. Mol Ecol 14: 2313-2318.

18. Yan-Ping WU, Guan WJ, Zhao QJ, Xiao-Hong HE, Ya-Bin PU, et al (2009) A fine map of maternal lineage analysis by mitochondrial hypervariable region in 12 chinese goat breeds. Anim Sci J 80: 372-380.

19. Vacca GM, Daga C, Pazzola M, Carcangiu V, Dettori ML, et al. (2010) Dloop sequence mitochondrial DNA variability of Sarda goat and other goat breeds and populations reared in the Mediterranean area. J Anim Breed Genet 127: 352-360.

20. Zhao Y, Zhang J, Zhao E, Zhang X, Liu X, et al. (2011) Mitochondrial DNA diversity and origins of domestic goats in Southwest China (excluding Tibet). Small Ruminant Res 95: 40-47.

21. Ahmed S, Grobler P, Madisha T, Kotzé A (2017) Mitochondrial D-loop sequences reveal a mixture of endemism and immigration in Egyptian goat populations. Mitochondrial DNA J Part A 28: 711-716.

22. Mdladla K, Dzomba EF, Muchadeyi FC (2018) Landscape genomics and pathway analysis to understand genetic adaptation of South African indigenous goat populations. Heredity 120: 369-378. 